\title{
Color Texture Segmentation Based on Quaternion-Gabor Features
}

\author{
Wang Xiao-Hui, Zhou Yue, Wang Yong-Gang, and Zhu WeiWei \\ Institute of Image Processing and Pattern Recognition, \\ ShangHai JiaoTong University, ShangHai, 200240, China \\ \{chocobowang, wygang\} @gmail.com, \\ \{zhouyue, zhuww $a$ sjtu.edu.cn
}

\begin{abstract}
This paper proposed a new framework for color texture segmentation which integrated the color and texture features. Quaternion-Gabor filter was first introduced in this paper for color texture segmentation. The algorithm achieved color texture multichannel Gabor filtering through DRBFT and IDRBFT. And the quaternion-Gabor filter extracted the input color image's color features and texture features at the same time. The proposed method was tested using different mosaic and natural images. Despite the simplicity of the whole algorithm, the segmentation results were rather encouraging.
\end{abstract}

Keywords: DRBFT, RBs, MCF, k-means.

\section{Introduction}

Sangwine introduced the first example of a holistic color image filter based on quaternion convolution in 1998 [1]. Although encouraging results have been achieved in color image processing based on quaternions, the multiplication rule of quaternions was not commutative, which restricted the applications of quaternions in signal and image processing. To address this problem, Pei et al. introduced reduced biquaternions (RBs) in digital signal and image processing in 2004 [2]. The multiplication rule of RBs was commutative. One of its advantages was that many existing conventional complex Fourier transform algorithm could be used to implement the discrete reduced biquaternions Fourier transform (DRBFT).

The multichannel filtering (MCF) approaches have appeared to be one of the most successful grey texture segmentation algorithms among all the grey texture segmentation methods. The problem of using MCF algorithm to process color image is how to implement the color image convolution. In this paper color images are represented and processed using RBs. With the DRBFT and corresponding IDRBFT algorithm, we can implement the color image holistic convolution successfully. Therefore, many existing Gabor filtering algorithms which have ever been used to process grey images can be extended to process color images [3][4]. In this paper the Converging Squares Algorithm (CSA) algorithm that has described in [4] is used to design the channels of the MCF. That is to say the number of channels and channel parameters are automatically determined from power spectrum of input color images. 
In this paper after the input images are filtered by MCF our method analyzes the color texture using the concept of textons. First we combine each pixel's filtering outputs' amplitudes and phases into a vector. Since the feature vectors are highly correlated, we use the principal components analysis (PCA) [7] to reduce the feature space's dimensionality. Then the k-means [6] method is adopted to cluster the reduced dimension's feature vector and then the region merging algorithm is employed to combine the adjacent clusters that achieved by the k-means method. At last if the texture edge is not very accurate, a special thresholding method will be adopted based on the mean grey value, where each pixel's grey level value is computed with a small neighborhoods (e.g. $7 \times 7$ ) around it.

The rest of this paper is organized as follows: the MCF algorithm and the RBs, DRBFT and IDRBFT are briefly introduced in Section 2 and Section 3 respectively. The principle of the proposed color texture segmentation algorithm is described in Section 4 and some experimental results are given in Section 5 to demonstrate the effectiveness of the proposed color texture analysis algorithm. Finally, we conclude this paper in Section 6 and present future work.

\section{Multichannel Filter Model (MCF)}

Bovik et al. proposed the multichannel texture filtering technique that relies on 2-D Gabor filters to isolate regions of perceptually homogeneous texture in an image [3]. A special case of the Gabor filter is the daisy petal filter, in which the filter lobes radiate from the origin of the spatial frequency domain [3] [4]. In this paper a bank of daisy petal Gabor filters is used to encode the input color images into multiple complex-modulated sub-images.

The continuous domain impulse response function of the daisy petal Gabor filter can be wrote as the following general form:

$$
h(x, y)=g\left(x^{\prime}, y^{\prime}\right) \cdot \exp \left[2 \pi j F x^{\prime}\right] .
$$

where $\left(x^{\prime}, y^{\prime}\right)=(x \cos \phi+y \sin \phi,-x \sin \phi+y \cos \phi)$ are notation of coordinates, major oriented at an angle $\phi$ from the $\mathrm{x}$-axis. $\mathrm{F}$ is radial centre frequency, and $j=\sqrt{-1}$.

The Gaussian component is

$$
g(x, y)=\left(\frac{1}{2 \pi \lambda \sigma^{2}}\right) \cdot \exp \left(-\frac{(x / \lambda)^{2}+y^{2}}{2 \sigma^{2}}\right) .
$$

where $\sigma$ is the Gaussian scale factor and $\lambda$ is the aspect ratio between the $\mathrm{x}$ and $\mathrm{y}$ axes. The spatial frequency response of the daisy petal Gabor filter (1) is

$$
H(u, v)=\exp \left\{-2 \pi^{2} \sigma^{2}\left[\left(u^{\prime}-F\right)^{2} \lambda^{2}+\left(v^{\prime}\right)^{2}\right]\right\} .
$$

Like the algorithm used in [4], the Converging Squares Algorithm (CSA) proposed by O'Gorman and Sanderson [5] is also used in this paper to detect the spectral peak and determine the channels parameters. 


\section{Reduced Biquaternions (RBs)}

In 2004 Pei et al. adopted RBs to represent and process color image [2]. The representation of RBs is

$$
q=q_{r}+q_{i} i+q_{j} j+q_{k} k=\left(q_{r}+q_{i} i\right)+\left(q_{j}+q_{k} i\right) j \equiv q_{a}+q_{b} j .
$$

where $q_{a}$ and $q_{b}$ are two complex numbers, $q_{r}, q_{i}, q_{j}$ and $q_{k}$ are real numbers, and $i, j, k$ obey the rules as follows:

$$
i j=j i=k, j k=k j=i, i k=k i=-j, i^{2}=k^{2}=-1, j^{2}=1 .
$$

Therefore, from (5) we can find that the multiplication of RBs is commutative. It is a unique advantage over the quaternions.

Similar to the quaternion, an RB can also be uniquely represented by a polar form if $\delta \neq 0$ :

$$
q=q_{r}+q_{i} i+q_{j} j+q_{k} k=A e^{i \theta_{i}} e^{j \theta_{j}} e^{k \theta_{k}}
$$

where $A=|q|=\sqrt[4]{\delta} \geq 0, \theta_{i} \in(-\pi, \pi], \theta_{j} \in R, \theta_{k} \in(-\pi / 2, \pi / 2]$, and the definition of $\delta$ and the proof of (5) can be found in [2].

In paper [2], the author defined two types' DRBFT. In this paper we use the DRBFT of type 1[2]:

$$
F_{(R B 1)}(p, s)=\sum_{m=0}^{M-1} \sum_{n=0}^{N-1} f(m, n) e^{-2 \pi\left(i \frac{p m}{M}+j \frac{s n}{N}\right)} .
$$

where $f(m, n)$ is a 2-D RBs signal, and $i^{2}=j^{2}=-1$. The RB Fourier transform can be implemented by decomposing the RBFT into a pair of conventional complex Fourier transform. So first step is to decompose the RB into following form [2]:

$$
f(m, n)=f_{a}(m, n)+f_{b}(m, n) j
$$

Then

$$
F_{(\mathrm{RB})}(p, s)=\operatorname{DFT}\left(f_{a}(m, n)\right)+\operatorname{DFT}\left(f_{b}(m, n) j\right) .
$$

Thus, two conventional complex 2-D DFTs can be used to implement the RBFT.

The definition of $\mathrm{RB}$ convolution is

$$
g(m, n)=f(m, n){ }_{R B} h(m, n)=\sum_{\tau=0}^{M-1} \sum_{\eta=0}^{N-1} f(m-\tau, n-\eta) h(\tau, \eta) .
$$

where ${ }_{\mathrm{RB}}$ is $\mathrm{RB}$ convolution.

The multiplication rule of RBs is commutative. Here we can get:

$$
g(m, n) \equiv \operatorname{IDRBFT}\left\{F_{(R B)}(p, s) H_{(R B)}(p, s)\right\}=f(m, n) *_{R B} h(m, n) .
$$




\section{The Proposed Color Texture Segmentation New Algorithm}

The principles of the proposed color texture segmentation algorithm can be better explained by referring to the block diagram given in Fig. 1. The whole algorithm is divided into eight successive steps: spectral peak detection, multichannel filtering, channel grouping, dimension reduction, k-means clustering, region merging, thresholding and post-processing.

Spectral peak detection, just as described in the preceding section, using the CSA algorithm determines the number of multichannel filtering channels and frequencies and orientations of each channel.

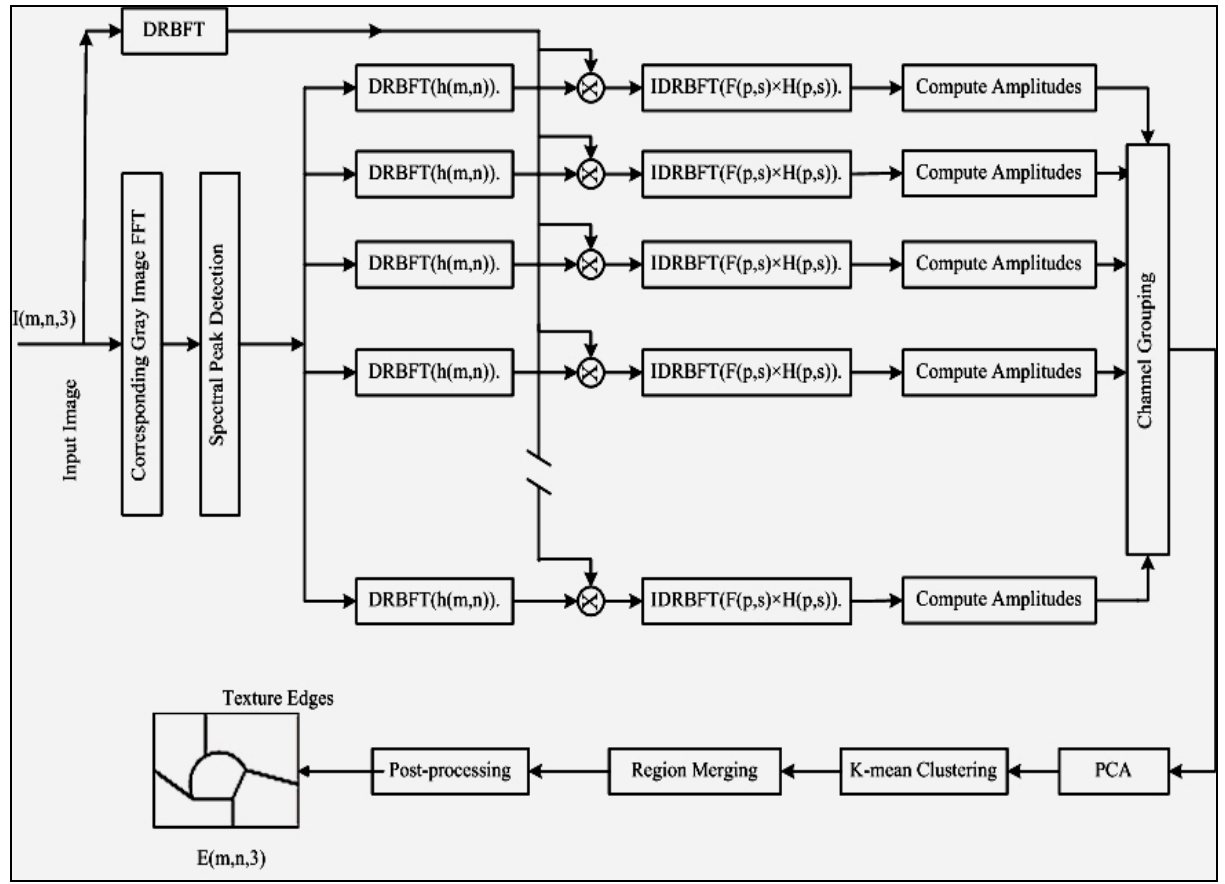

Fig. 1. Block Diagram of the Proposed Algorithm

Then the next step is multichannel filtering. To computer the convolution between the input color image and Gabor filter kernel, the input color image and the Gabor kernel are expressed using RBs firstly. Then we can calculate the RBBDT of the input color image and the Gabor kernel. At last the convolution between the input color image and Gabor filter kernel of each channel can be calculated using (11).

After getting the each channel's filtering result, we compute the amplitudes and phases of each channel's output. And then the amplitudes and phases of each pixel are 
combined to a feature vector. Just as former explanations, the filtering output of each channel is a RB matrix. So each pixel has the form of formula (4). For each channel we compute three amplitudes of the filtering result. We use (6) to get the first amplitude. And we look on the first two parts of RB: $q_{r}+q_{i} i$ and the last two parts of RB: $q_{j}+q_{k} i$ as two general complex numbers, so we can use the general definition of a complex number's amplitude compute the other two amplitudes. Through experiments, we find that using three amplitudes can gain much more edge information of the input color image. Therefore, in this paper three amplitudes are used rather than one which can provide better discriminating power. From (6), we can compute three phases from each channel's output. And the three phases can also be used to identify the input color image's edge. Then we combine each pixel's filtering outputs' amplitudes and phases into a vector. Therefore, each pixel $\left(m_{i}, n_{j}\right)$ is now represented by an $n$-dimensional feature vector.

Since the extracted feature vectors are highly correlated, we use the principal components analysis (PCA) [7] to reduce the feature space's dimensionality. Then the following stage is to classify each image pixel using the k-means algorithm in terms of the each pixel's feature vector. This segmentation is often referred to the conception of textons. From the output of the k-means clustering, we can get the each pixel's rough classification information.

Then a region merging method is used to combine adjacent clusters which are statistically similar. The initial clustering is based on k-means cluster with $k$ set to be larger than the anticipated number of final regions. As a result, after the initial clustering some regions still need to be merged. The region merging is done in an agglomerative manner where in each iteration the two most similar regions are merged. The similarity of two regions $R_{i}$ and $R_{j}$ is given by

$$
\zeta_{i, j}=\left(u_{i}-u_{j}\right)^{T}\left[\sum_{i}+\sum_{j}\right]^{-1}\left(u_{i}-u_{j}\right)
$$

where $u_{i}, u_{j}$ are the mean vectors and $\sum_{i}, \sum_{j}$ are the covariance matrices computed from the feature vectors of regions $R_{i}$ and $R_{j}$ respectively. Here we use the region similarity measure analogous to the one proposed in Nguyen et al.[8].

The last stage is obtaining the segmentation image from the region merging result by a special thresholding method. Each image pixel's thresholding value is determined in terms of average grey level value of $w \times w$ window centered on it. Finally from the thresholding image, the color texture edge can be detected and superimposed on the original color texture image.

\section{Segmentation Results}

The proposed algorithm has been tested extensively with a variety of synthetic and natural color textures. And the segmentation results using the proposed algorithm are compared with JSEG (J measure based SEGmentation according to [9]) algorithm. 
The color textures used in this paper are mainly obtained from the VisTex color texture images database [10] and the Berkeley Segmentation Dataset [11]. The VisTex texture images and Berkeley Segmentation Dataset are both available to the public from their web sites.

Fig. 2 shows the segmentation results of mosaic color images. Owing to only color feature can be used to segment, the JSEG algorithm can not obtain good segmentation result. Fig. 2(a) shows the JSEG algorithm can not identify the color texture because there is only texture feature of different orientation that can be used to segment. Fig. 2(b)(c) show the JSEG algorithm over segment the input images. Fig. 2(d)(e)(f) show the segmentation results of the proposed algorithm. The results are rather good because of the proposed algorithm can adopt the input image's color features and texture features to segment.

The segmentation results of natural color images are shown in Fig. 3. The segmentation results of the JSEG algorithm are showed in Fig. 3(a)(c)(e)(g). Because natural images have enough color features, the segmentation results of the JSEG algorithm are very good. The segmentation results of the proposed algorithm are showed in Fig. 3(b)(d)(f)(h). Not that all the important objects in the images are well identified. The segmentation results are nearly close to or even exceed the segmentation results of JSEG.

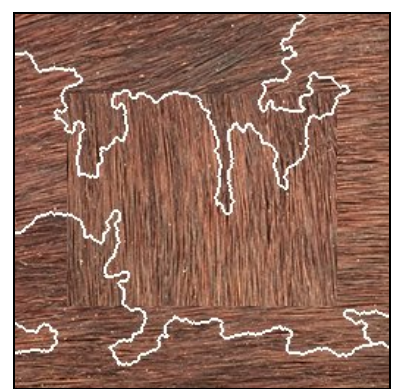

(a)

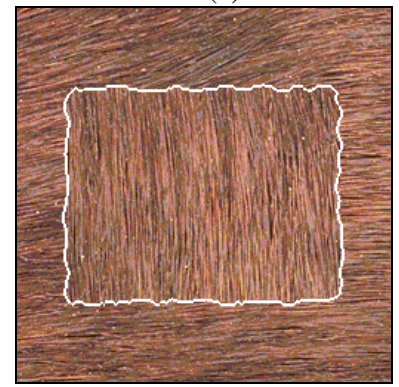

(d)

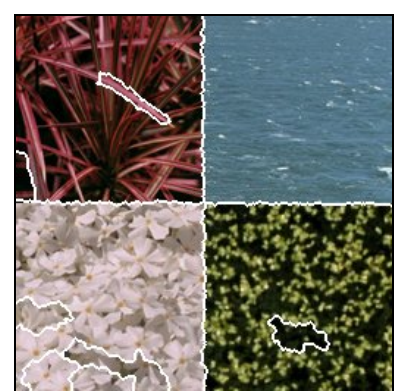

(b)

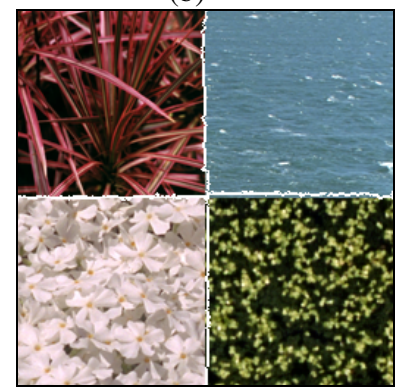

(e)

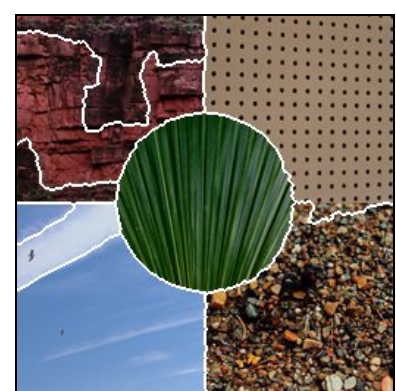

(c)

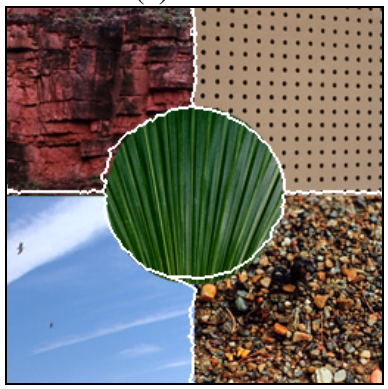

(f)

Fig. 2. Segmentation results of mosaic images. (a) (b) (c) Segmentation results using the JSEG algorithm (d) (e) (f) Segmentation results using proposed algorithm. 


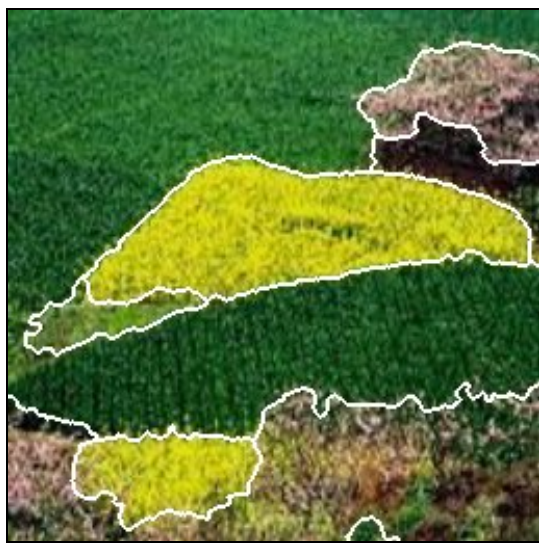

(a)

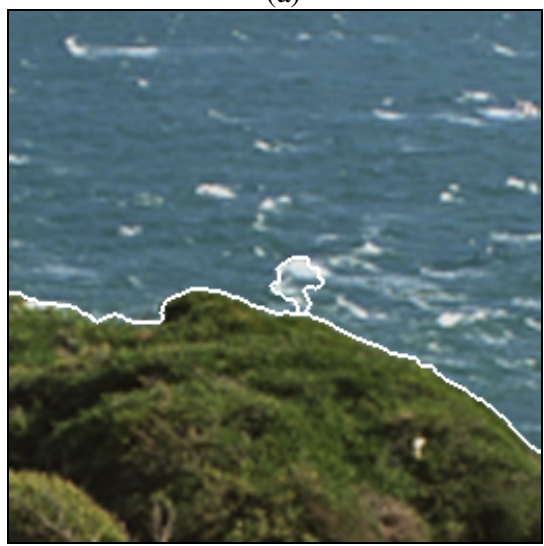

(c)

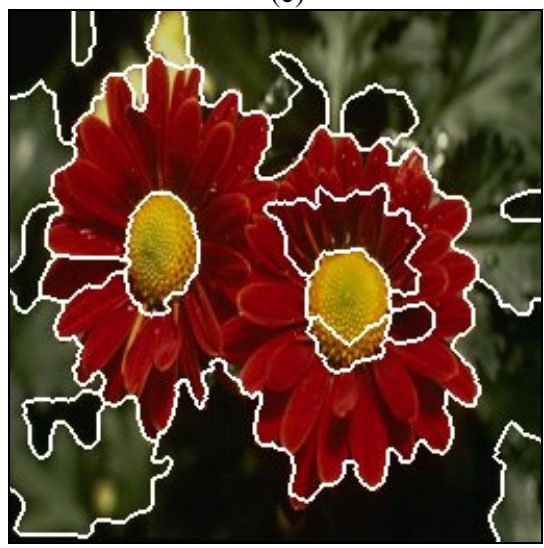

(e)

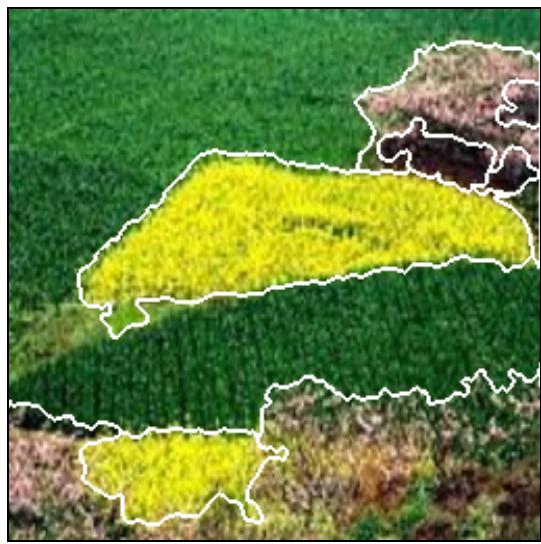

(b)

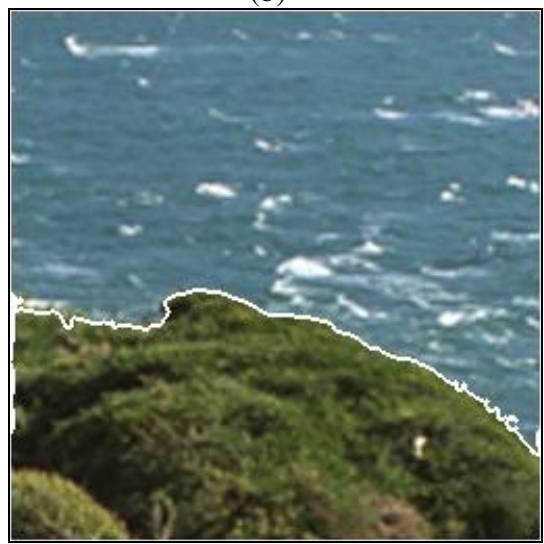

(d)

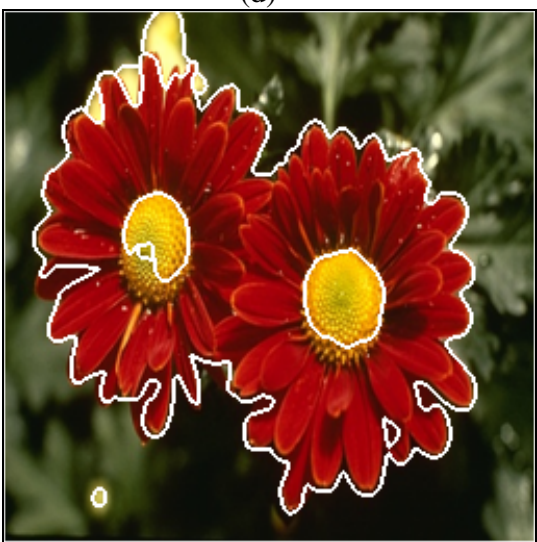

(f)

Fig. 3. Segmentation results of some natural images. (a) (c) (e) (g) Segmentation results using the JSEG algorithm and (b) (d) (f) (h) segmentation results using the proposed algorithm. 


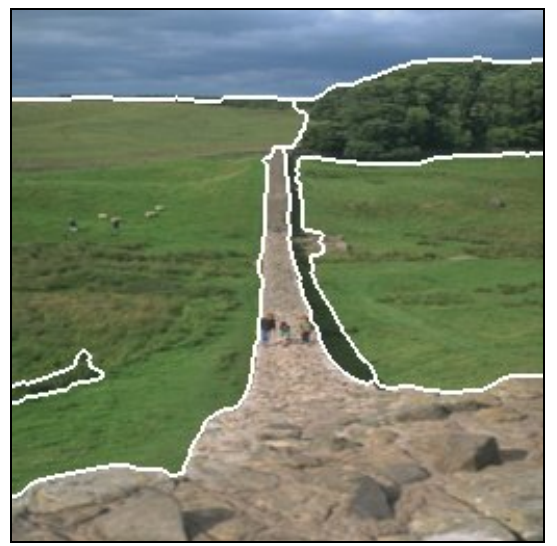

(g)

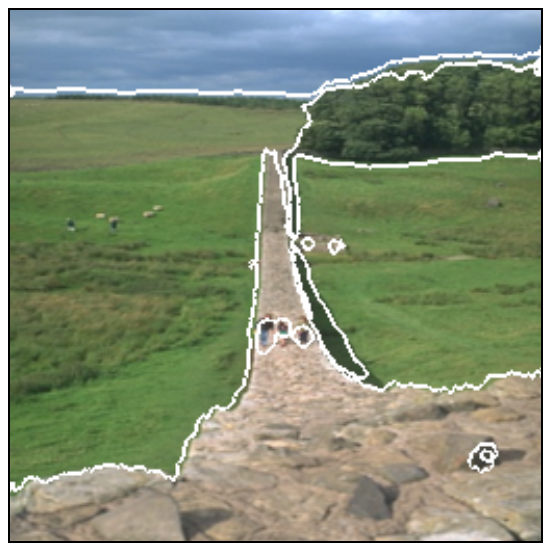

(h)

Fig. 3. (continued)

\section{Conclusions}

A new framework was developed for color texture segmentation which integrated the color and the texture features. The contribution of color features and contribution of texture features were both used for color texture discrimination. And the quaternionGabor filter extracted the input color image's color features and texture features at one time. The method was applied to various mosaic and natural images. And the segmentation results obtained showed a remarkable segmentation. In conclusion, color texture segmentation and other color image processing techniques based on quaternions will become more and more attractive. In the future work, we plan to find method to fix the $\mathrm{k}$ in k-means automatically.

\section{References}

1. S. J. Sangwine, "Color image edge detector based on quaternion convolution," Electronics Papers, Vol. 34 No. 10, pp.969-971, May, 1998.

2. Soo-Chang Pei, Ja-Han Chang, and Jian-Jiun Ding, "Commutative reduced biquaternions and their Fourier transform for signal and image processing Applications," IEEE Transactions on Signal Processing, Vol. 51, No. 7, pp.2012-2031, July, 2004.

3. A. C. Bovik, M. Clark and W. S. Geisler, "Multichannel texture analysis using localized spatial filters," IEEE Transactions on Pattern Analysis and Machine Intelligence, No. 12, pp.55-73, 1990.

4. T. N. Tan, "Texture Edge Detection by Modelling Visual Cortical Channels", Pattern Recognition, Vol.28, No.9, pp.1283-1298, 1995.

5. L. O'Gorman and A. C. Sanderson, "The converging squares algorithm: an efficient method for locating peaks in multidimensions," IEEE Transactions on Pattern Analysis and Machine Intelligence, Vol.6, No. 3, pp.280-288, 1984.

6. A. K. Jain and R. C. Dubes, Algorithms for clustering data. Prentice Hall, Advanced Reference Series, New Jersey, 1988. 
7. M. Kirby, F. Weissor, G. Dangelmayr, "A model problem in the representation of digital image sequences," Pattern Recognition, Vol. 26, No.1, pp.63-73,1993.

8. H. Nguyen, M. Worring, A. Dev, "Detection of moving objects in video using a robust motion similarity measure," IEEE Transactions on Image Processing, Vol.9, pp.137-141, 2000.

9. Y.Deng, and B.S.Manjunath, "Unsupervised segmentation of color-texture regions in images and video," IEEE Transactions on Pattern Analysis and Machine Intelligence, Vol. 23, No.8, pp.800-810, Aug., 2001.

10. VisTex. http://vismod.media.mit.edu/vismod/imagery/VisionTexture/vistex.html

11. Berkeley Segmentation Dataset. http://www.eecs.berkeley.edu/Research/Projects/CS/ vision/grouping/segbench/ 